\title{
Colposcopic genital findings in prepubertal girls assessed for sexual abuse
}

\author{
C J Hobbs, J M Wynne, A J Thomas
}

\begin{abstract}
After the introduction of the colposcope for the examination of children suspected of being sexually abused in Leeds, a study was undertaken to described the findings in detail. A total of 109 consecutive prepubertal girls, mean age 70.4 months were assessed including colposcopic genital and anal examination, and peer group review of reports and photographs. Fifty nine children had signs consistent with blunt force penetrating trauma (hymenal transection/major notch, scar, or hymenal attenuation). Transections were encountered most commonly at 6 o'clock (directly posterior).

In 46 the hymenal orifice was gaping with thigh abduction only and in 47 the hymenal orifice transverse diameter was greater than $4 \mathrm{~mm}$ on labial separation.

Overall, physical findings were commonly present and in only two cases were no signs recorded. Non-specific and frequent findings included patterns of labial and introital reddening. Supportive hymenal signs including swelling, rounding of edge, thickening, distortion, and loss of symmetry were common. Labial fusion was present in 20 . Urethral dilatation with labial separation was noted in 14.

Physical findings including normality are consistent with abuse and even minor anogenital signs as well as negative findings should be documented. Colposcopy and photography are valuable tools in peer review, teaching, and case management.
\end{abstract}

(Arch Dis Child 1995; 73: 465-471)

Keywords: colposcope, sexual abuse, genital findings, prepubertal girls.

Department of
Community
Paediatrics, St James's
University Hospital,
Beckett Street, Leeds
LS9 7TF
C J Hobbs
A J Thomas
Leeds General
Infirmary
J M Wynne
Correspondence to:
Dr Hobbs.

Child sexual abuse (CSA) has received increased public attention since the Cleveland inquiry in 1987,1 and genital findings in prepubertal girls who have been sexually abused have been the subject of a report of the Royal College of Physicians. ${ }^{2}$

Increasing numbers of cases referred have encouraged the formation of teams of trained paediatricians to undertake medical examinations. In Leeds more doctors are performing these examinations. Research in the UK and
US has provided information about the genital findings in groups of abused children and children selected for non-abuse. Increasing consensus exists on the significance of genital appearances in prepubertal girls. The colposcope, widely used in the US for this work, is increasingly available in the UK.

Referrals for all forms of abuse to paediatricians in Leeds in 1993 included 292 children diagnosed as confirmed or probable cases of CSA. Profiles of children from cohorts in $1985 / 6$ and 1989 were described in earlier publications. ${ }^{34}$ Both cohorts contained $72 \%$ girls and $28 \%$ boys with the mean age 8.0 years $^{3}$ (38\% under 5 years) and 6.9 years $^{4}$ ( $41 \%$ under the age of 5 years) respectively. Referral patterns have remained the same during the period of the present study.

The aim of the study was to describe the genital findings in prepubertal females with suspected CSA. No attempt has been made to correlate the findings with any history, disclosure, or other parameter of abusive experience.

\section{Methods}

Urgent and non-urgent cases are referred for medical examination to paediatricians from various agencies including social services, police, education (schools and nurseries), and various primary and secondary health care providers. Clinical presentation includes: a disclosure by the child, witness, or perpetrator of the abuse; physical, behavioural, and psychosomatic symptoms; and evidence of other abuse or neglect of the child including nonaccidental injury. ${ }^{5}$

Siblings are routinely examined and other children known to have been in contact with a suspected abuser.

In 1991, following others' experience, 67 the colposcope was introduced in Leeds for anal inspection in both sexes and genital inspection in girls. Trained examiners used Olympus OCS1, OCS2, and OCS3 colposcopes. The colposcope provides variable magnification, a cold light source, and integral $35 \mathrm{~mm}$ colour photography producing images of high quality. Slides were obtained using Kodak Ektachrome $160 \mathrm{~T}$ film.

After a period of familiarisation with the instrument, a study was designed to collect information on the genital and anal findings in 
Physical signs in 107 children with concerns regarding CSA

Erythema labia
Erythema vestibule
Localised erythema (symmetrical)
Localised erythema (asymmetrical)
All erythema
Abrasion labia
Abrasion vestibule
Bruise labia
Bruise vestibule
Discharge
Wart
Friable fourchette
Tear fourchette
Posterior midline avascular area
Labial adhesion (extent 2-12 mm, mean
$\quad 6$ mm)
Hymenal orifice visible
Hymenal orifice gaping
Hymenal orifice not opening
Hymenal orifice transverse diameter
$\quad \leqslant 4$ mm
Hymenal orifice transverse diameter
$>4$ mm
Hymenal orifice transverse diameter
unmeasured
Hymen attenuation
Abrasion hymen
Contusion hymen
Swelling hymen

\begin{tabular}{lr}
\hline Thickening of hymenal edge & 50 \\
Rounding of hymenal edge & 31 \\
Distortion of hymenal rim including & \\
bumps & 64 \\
Hymen transection/deep notching & 50 \\
Hymen transection/notch position & \\
12 o'clock & 3 \\
Position 6 o'clock & 25 \\
Position $11 / 1$ o'clock & 8 \\
Other position & 25 \\
Hymen loss of symmetry & 62 \\
Adhesions: hymen to surrounding & \\
structures & 5 \\
Hymenal remnants & 6 \\
Hymen: decreased vascularity & 9 \\
Hymen: increased vascularity & 0 \\
Hymen septum & 13 \\
Vaginal ridge & 1 \\
Vaginal abrasion & 3 \\
Vaginal erythema & 12 \\
Urethral swelling & 14 \\
Urethral dilatation & 2 \\
Periurethral bands & \\
Mean (mm) hymenal orifice transverse & $4 \cdot 23$ \\
diameter age 20-59 months (29) & $4 \cdot 23$ \\
Mean (mm) hymenal orifice transverse & $4 \cdot 26$ \\
diameter age $60-99$ months (35) & $4 \cdot 26$ \\
Mean (mm) hymenal orifice transverse & $6 \cdot 17$ \\
diameter age 100 months plus (18) & $6 \cdot 17$ \\
\hline
\end{tabular}

a group of children where sexual abuse was suspected. Consecutive prepubertal girls referred with concerns regarding CSA examined and photographed by the colposcope were studied.

A full paediatric assessment and clinical examination was performed by a single examiner. The genital examination was undertaken with the child at the end of an examination couch in the supine frog-legged position. The colposcope head (with extension lens) was positioned $300 \mathrm{~mm}$ (12 inches) from the perineum. Anal examination, not reported here, was undertaken in the left lateral position with buttocks separated.

Photographs were taken at various magnifications $(7 \times$ to $21 \times)$ after verbal consent of the parent, and child where appropriate, had been obtained. The camera was operated by remote foot switch.

Hymenal opening and relaxation was achieved by either labial separation or labial traction. Transverse and vertical (anteroposterior) hymenal opening diameters were measured using short lengths of Perspex rule or tape measure held just in front of the hymen to avoid parallax error. Anteroposterior diameter measurements are not included because of difficulties of precise measurement. Hymenal diameter varies with the method of examination used. ${ }^{8}$ Measurements were made using labial separation.

Initial assessment of the genitalia was made with thigh abduction without labial separation. The presence of a visible hymenal orifice (described as gaping) in this situation was noted.

Routine examination in the knee-chest position as commonly practised in North America was not used. Medical examinations unfortunately are not always supported by professional colleagues or parents in this country. Adding a further (undignified) routine examination position to the procedure could increase resistance. However, the position was occasionally used to visualise the anatomy of the posterior hymen.

Cases were excluded if adequate photographs were not available.
Repeat examination frequently formed part of ongoing assessment and follow up in cases with or without court direction. These examinations may assist interpretation retrospectively of earlier findings. Photographs, case notes, and medical reports were discussed at monthly peer review meetings.

Findings were recorded on a protocol of 46 genital and 28 anal signs with known association with CSA. Wider information about the case contributing to the diagnostic jigsaw of $\mathrm{CSA}^{9}$ was discussed and cases allocated into one of three categories: suspected, probable, or confirmed.

A suspected case included one where there were grounds for concern insufficient for child protection procedures to be commenced. These cases were usually followed up while continuing to discuss with the parents and professional colleagues diagnostic options including CSA.

A probable case was one where a summation of findings was sufficiently worrying, in the view of the paediatricians, to warrant immediate or continued involvement of social agencies for the development of a specific plan of investigation. ${ }^{4}$ Examples included children with emotional or behavioural indicators of sexual abuse or less than gross physical signs or an infection known to be sexually transmitted in some cases.

A confirmed case was one where the child provided a clear disclosure of abuse, or a perpetrator admitted the offence, or where gross physical signs of genital or anal trauma without other reasonable explanation were present or a sexually transmitted disease known to be sexually transmitted in virtually every case was present. ${ }^{4}$

\section{Results}

One hundred and nine prepubertal girls with a mean age of 70.4 months (range 12-124 months) were included. There were 18 suspected, 52 probable, and 39 confirmed cases. In two children entirely normal findings were present; 107 children exhibited one or more findings as detailed in the table.

Genital erythema was very commonly present and on its own is a finding with low specificity for abuse. It was predominantly labial, introital, or both. A common pattern was inner surface reddening of the labia minora. Bilateral symmetrical reddening of the labia majora in a distinct vertical band is sometimes referred to as 'tramline reddening'. A more unusual pattern was patchy, asymmetri$\mathrm{cal}$, and uneven reddening which may reflect localised injury.

Acute signs consistent with injury were commonly seen. Swelling in 33 in the absence of discharge or signs indicating infection is evidence of injury. Follow up of this sign is important as other evidence of injury may become visible as swelling settles. The diameter of the hymenal orifice may change as swelling subsides.

Bruising, abrasions, and acute tears of labia, vestibule, and hymen were less commonly 


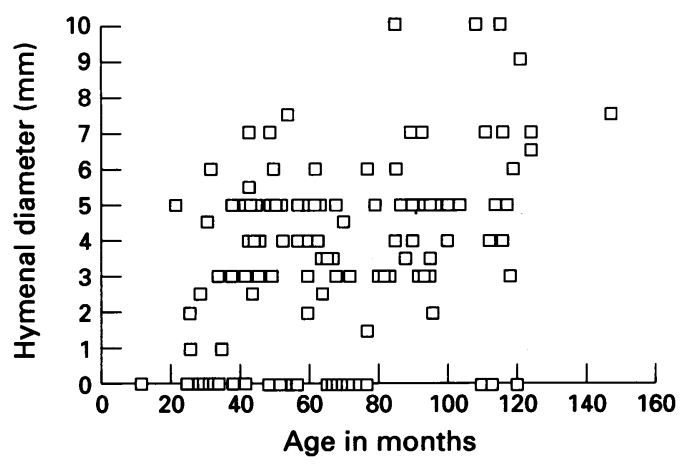

Transverse hymenal diameter plotted against age.

seen. The colposcope facilitates detection and accurate diagnosis of abrasions.

Discharge was present in nine children. Fine wire 'ear, nose, and throat' swabs for isolation and culture of sexually transmitted diseases can be carefully inserted into the vagina through the hymenal opening under colposcope observation without causing discomfort. In none of these cases was a sexually transmitted infection isolated. One child had anogenital warts.

Abnormalities of the posterior fourchette are associated with sexual abuse. Scars can be confused with midline avascular areas which are probably normal. These were found in nine cases. In one a tear was present and in six fourchette friability lead to slight bleeding after separation of the superficial layer of the skin when the labia were separated.

There were 20 with labial fusion in whom mean age of 60 months was less than the group as a whole (70.4 months). With the colposcope, posterior labial fusion of 1 or $2 \mathrm{~mm}$ is readily visible. The maximum extent of the fusion was $12 \mathrm{~mm}$ with a mean of $6 \mathrm{~mm}$.

'The size of the hymenal orifice has been the subject of a great deal of discussion and disagreement, the most commonly held view being that an orifice after labial separation with transverse diameter greater than $4 \mathrm{~mm}$ in the prepubertal child is strongly correlated with abuse'. ${ }^{2}$ The transverse hymenal orifice diameter was measured in 82 girls and was greater than $4 \mathrm{~mm}$ in 45 and $4 \mathrm{~mm}$ or less in 37.

The relationship between transverse hymenal diameter measured with labial separation and age is shown in the figure. No clear relationship is evident up to 100 months. The mean diameter in the group 100 months and over was significantly greater than the other groups 20-59 and 60-99 months which were the same. The range of transverse hymenal diameter was $1 \mathrm{~mm}$ to $10 \mathrm{~mm}$. All three measuring $10 \mathrm{~mm}$ were aged between 90 and 120 months.

At all ages a minority of hymenal orifices were difficult to open by labial traction or separation. Follow up may distinguish normal cases from those with secondary obliteration with distorted anatomy after inflammation, the result of trauma, or infection. ${ }^{10}$

In 46 the hymenal orifice was gaping. A view into an open vagina between partially separated labia majora was obtained with the child in the frog-leg position. The mean transverse hymenal diameter where gaping was present was $5.2 \mathrm{~mm}$ as opposed to $4.0 \mathrm{~mm}$ where the orifice opened but did not gape (non-gaping). Hymenal attenuation was present in $42 \%$ and $13 \%$ and transection in $56 \%$ and $46 \%$ of gaping and non-gaping respectively. Mean age of the groups was $77 \cdot 6$ and 68.7 months respectively.

Hymenal laceration, scars, and attenuation are considered diagnostic of blunt force penetrating trauma. ${ }^{2}$ Attenuation was present in 27, transection or major notch in 50, and scars in four with 59 having one or more of these signs.

The site of hymenal transection or major notch was directly posterior or 6 o'clock in 25 , directly anterior or 12 o'clock in three, and in eight either at 11 or 1 o'clock. In 25 the transection or major notch was in one of the remaining positions ( 7 to 10 and 2 to 5 o'clock). In 12 children more than one of these four sites were involved.

Posterior transections often appear as a deep V-like cleft extending into the posterior hymen with sharp angle rather than smooth curve at 6 o'clock. Anterior hymenal transections are considered more difficult to recognise because of the normal shape of the crescentic hymen which extends bilaterally and symmetrically in the 11 and 1 o'clock positions. However, injury can extend further anteriorly either symmetrically or asymmetrically leading to deficient hymen in this site.

Unusual urethral findings included swelling in 12, dilatation of the orifice on labial separation in 14, and periurethral bands in two. Six cases exhibited both swelling and dilatation. The mean age of those with dilatation was $61 \cdot 7$ months. All had open hymenal orifices (mean diameter $4.35 \mathrm{~mm}$ ), four of which gaped.

Vaginal ridges were found in 13 and are recognised as normal anatomical findings. Abnormal vaginal findings were less commonly seen with abrasion in one, and erythema in three.

\section{Discussion}

Children continue to be referred by agencies in Leeds who have a decade of experience of work in CSA and longer in physical abuse. The paediatrician's experience is that many of these children will have been abused and this study supports that experience. Multidisciplinary work has enabled the development of a jigsaw approach to diagnosis in CSA where the medical examination is an important and essential part. ${ }^{9}$ Many children at the time of medical examination and investigation have not made disclosures, although some do during the investigation or later when protected. In 1989 Frothingham et al found that $35 \%$ diagnosed as confirmed or probable cases of CSA continued to be abused while the plan for their protection was being developed. ${ }^{4}$ The findings from this study indicate that the paediatrician is frequently able to contribute information of diagnostic importance from the genital examination to the overall picture. What it is not appropriate for the paediatrician 
to do is either to confirm or dismiss allegations of CSA in order to meet the demands of criminal investigation or to satisfy the needs for corroboration of the child's story. ${ }^{11}$

Genital findings in normal 'non-abused' children and after CSA have been the subject of a number of reviews. ${ }^{211-20}$ Studies in this area fall into those of newborns, 'non-abused' populations, ${ }^{131618}$ and clinical samples of children suspected or confirmed to have been abused. 121415172021

There are problems with all studies of normality or those selected for non-abuse because it is inevitable that some abused children will be inadvertently included. However, studies have attempted to reduce this risk. ${ }^{13} 1618$

Various findings from this study require discussion in the light of other work. Labial adhesions are usually diagnosed in the preschool child and they spontaneously resolve during childhood. Very minor degrees of superficial fusion are common in infancy with examination by the colposcope. ${ }^{1316}$ In studies of 'non-abused' children over the age of 12 months, labial fusion over $2 \mathrm{~mm}$ in length is less common. Thick and extensive fusion is said to be more likely to be associated with trauma. ${ }^{11}$ In this series $18 \%$ had labial fusion and this is comparable with other studies of sexually abused children. ${ }^{1522}$

Configuration of the hymen depends on age. The thicker redundant hymen of infancy is replaced by a thinner structure whose configuration includes posterior rim (crescentic) and annular variants. These different hymenal configurations have been considered in assessing the cases but not included in the descriptions. When oestrogen concentrations increase at puberty the hymen thickens and the appearances change markedly. These changes are obvious and children demonstrating them are not included here.

Much attention has focused onto hymenal orifice measurements. The opening can be measured in both transverse (horizontal) and anteroposterior (vertical) diameters but if the hymen is of posterior rim configuration, that is deficient anteriorly, it may be difficult to find a suitable place from which to measure the anteroposterior diameter. Anteroposterior measures have been omitted from this study.

In the studies of 'non-abused' children, examination position, degree of relaxation (including anaesthesia), and age affected the results. ${ }^{11}$ Labial traction gives the largest transverse measurements. The colposcopes used in this study do not incorporate integral eyepiece measuring devices. Therefore labial separation has been used with an external measuring device.

A clear relationship between age and transverse hymenal diameter was not present in this study between 20-99 months, unlike other studies, ${ }^{813}$ which have shown increasing diameter with increasing age. The results suggest that a gaping hymenal orifice is more likely to be related to a hymen that is attenuated and has a wider orifice even allowing for the greater mean age of the gaping group. The data support the view that this sign is important as a supportive sign of CSA, indicating that penetration may have occurred. It is less clear whether children selected for non-abuse also demonstrate this appearance.

The most commonly held view with regard to transverse hymenal diameter is that a diameter $>4 \mathrm{~mm}$ in the prepubertal child is associated with CSA. ${ }^{2}$ The results of the present study are consistent with the findings of previous studies supporting this view. ${ }^{19-21}$

Findings of major importance in diagnosing penetrative injury to the hymen are the presence of a transection, a hymenal scar, and attenuation or loss of hymenal tissue. A transection or tear in the hymen heals quickly and may result in either a normal appearance, an indentation, a notch, or major deficit in the hymen. It is important to be sure when examining the hymen that only fixed irregularities in various examination positions and degrees of relaxation are described. Sometimes cotton wool buds can be used to tease out suspicious areas or knee-chest examination performed to stretch a loose posterior hymen.

In this study, minor bumps associated with vaginal ridges and tags have not been described as there is good evidence for their normality. However, major and fixed notches (often with adjacent bumps) particularly associated with asymmetry, evidence of widening of the hymenal opening and a positive history from the child of painful penetration, have been included to indicate a previous and healed transection. The possibility that such notches represent a congenital finding remains, although posterior hymenal notches (6 o'clock) appear to be very unusual in 'nonabused' samples. ${ }^{13}$ This was the commonest site for a notch in this study, present in 25 children. In contrast anterior notches have been described in normal children but must also occur in CSA. Asymmetry of the hymen and increased diameters would add to concerns about this area.

Scars of the hymen are unusual and seen in only four children. Attenuation of the hymen (actual loss of hymenal tissue as the result of a traumatic process ${ }^{23}$ ) is thought to follow chronic abuse when the hymen is rubbed away. When gross, the hymen appears as a thin rim of tissue. Lesser degrees require a consideration of the thickness of hymenal tissue and the evenness of its distribution. Attenuation is frequently associated with a wide hymenal orifice and in this study with a gaping orifice. Thickening, asymmetry, and rounding of the hymenal edge are also thought to be associated with CSA. It was unusual in these children to encounter the fine lacy or wispy hymen well demonstrated in some texts. ${ }^{13}$ Obviously care must be taken when examining the genitalia not to distort the symmetry of the tissues.

Changes in the appearance of the urethral orifice are findings increasingly discussed in relation to CSA. ${ }^{3}$ It is probably important to specify the method of examination in assessing their significance. There is no agreed definition of dilatation. The appearances of the urethra vary considerably. Here 'dilatation' describes a urethra where a distinct open orifice exists on 
labial separation. The degree of dilatation can extend up to several millimetres and confusion with the vaginal orifice is possible. In our experience using labial separation, the urethral orifice usually appears closed.

There is less information available from the literature regarding normal and abnormal urethral orifice appearances. Gardner reports that urethral dilatation occurred in $28 \%$ of her sample of 79 prepubertal girls presumed nonabused, using labial traction. ${ }^{18}$ McCann and colleagues reported in a sample of 93 girls selected for 'non-abuse' aged 10 months to 10 years that $15 \%$ showed urethral dilatation with labial traction. ${ }^{16}$ However, it is also known that objects can be threaded into the urethra and even postpubertal urethral coitus has been described. ${ }^{24}$

Follow up of a 4 year old known to have been abused with urethral dilatation and abnormal genital and anal signs, showed fluctuating dilatation until she was protected. Further information is needed on this physical sign.

The colposcope has proved to be acceptable to children, parents, and doctors and is being used routinely in Leeds for examination in CSA. A more comfortable and detailed examination with ease of photographic recording are the main advantages. Photographs allow discussion with peers and can be used for teaching, audit, and research. Second medical opinions can be given without the need to re-examine the child. Changes on follow up examination can be better appreciated. Some examiners feel that the colposcope may not allow any more to be seen than with simple magnification by the otoscope or illuminated hand lens. ${ }^{12}$ Peer review allows objective assessment of complex information outside the tensions of the consulting room. Doctors are supported in discussing a difficult diagnosis usually with major implications for child, family, and professionals.

In conclusion, this study of children seen with concerns regarding CSA has demonstrated the effective use of the colposcope in the examination of the genitalia in prepubertal girls. All findings should be carefully documented. Interpretation of findings is made in the wider context of the history and previous examinations.

There is now greater consensus over the significance of physical signs in CSA. An association between urethral dilatation and a gaping hymenal orifice and CSA is suggested from this study. Other findings associated with CSA in other studies have been confirmed.

We thank Keymed for their continuing and generous support with colposcopes. The following colleagues have regularly with colposcopes. The following colleagues have regularly attended peer reviews and supported this work: L Allison, J
Clarke, P Gorham, F Lawrenson, S Lee, J Robertson, and S Wyatt.

1 Butler-Sloss E. Report of the inquiry into child abuse in Cleveland 1987. London: HMSO, 1988.

2 Royal College of Physicians. Physical signs of sexual abuse in children. A report of the Royal College of Physicians. London: RCP, 1991

3 Hobbs CJ, Wynne JM. Child sexual abuse - an increasing rate of diagnosis. Lancet 1987; ii: 837-41.

4 Frothingham TE, Barnett RAM, Hobbs CJ, Wynne JM. Child sexual abuse in Leeds before and after Cleveland. Child Abuse Review 1993; 2: 23-34.
5 Hobbs CJ, Wynne JM. The sexually abused battered child. Arch Dis Child 1990; 65: 423-7.

6 Teixeira RG. Hymenal colposcopic examination in sexual offences. Am F Forensic Med Pathol 1981; 2: 209-14.

7 Woodling BA, Heger A. The use of the colposcope in the diagnosis of sexual abuse in the pediatric age group. Child Abuse Negl 1986; 10: 111-4.

8 McCann J, Voris J, Simon M, Wells R. Comparison of genital examination techniques in prepubertal females. genital examination techniqu

9 Hobbs CJ, Wynne JM, Hanks HGI. Child abuse and neglect a clinician's handbook. Edinburgh: Churchill Livingstone, 1993.

10 Berkowitz CD, Elvik SL, Logan MA. A simulated 'acquired' imperforate hymen following the genital trauma of sexual abuse. Clin Pediatr (Phila) 1987; 26: 307-9.

11 Hobbs CJ, Wynne JM. The evaluation of child sexual abuse. In: Hobbs CJ, Wynne JM, eds. Balliere's Clinical Paediatrics. Vol 1. London: Bailliere Tindall, 1993: 1-29.

12 Muram D, Elias S. Child sexual abuse - genital tract findings in prepubertal girls. II comparison of colposcopic and unaided examinations. Am $\mathcal{F}$ Obstet Gynecol 1989; 160: 333-5.

13 Berenson AB, Heger AH, Hayes JM, Bailey RK, Emans SJ. Appearance of the hymen in prepubertal girls. Pediatrics 1992; 89: 387-94.

14 Herman-Giddens ME, Frothingham TE. Prepubertal female genitalia: examination for evidence of sexual female genitalia: examination for

15 Emans JS, Woods ER, Flagg NT, Freeman A. Genital findings in sexually abused, symptomatic and asymptomatic, girls. Pediatrics 1987; 79: 779-85.

16 McCann J, Wells R, Simon M, Voris J. Genital findings in prepubertal girls selected for non-abuse: a descriptive study. Pediatrics 1990; 86: 428-39.

17 Muram D. Child sexual abuse - genital tract findings in prepubertal girls. Am f Obstet Gynecol 1989; 160: 333-5.

18 Gardner JJ. Descriptive study of genital variations in healthy, nonabused premenarchal girls. I Pediatr 1992; 120: 251-7.

19 Cantwell HB. Vaginal inspection as it relates to child sexua abuse in girls under thirteen. Child Abuse Negl 1983; 7: 171-6.

20 Cantwell HB. Update on vaginal inspection as it relates to girls under thirteen. Child Abuse Negl 1987; 11: 545-6.

1 Heger A, Emans SJ. Introital diameter as the criterion for sexual abuse. Pediatrics 1990; 85: 222-3.

22 Berkowitz CD, Elvik SL, Logan MA. Labial fusion in prepubescent girls: a marker for sexual abuse? Am $\mathcal{f}$ Obstet pubescent girls: a marker

23 Emans SJ. Sexual abuse in girls: what have we learned about genital anatomy? ₹ Pediatr 1992; 120: 258-61.

24 Shikla VK, Tripathi VNP. Urethral coitus. Urology 1982; XIX: $542-3$.

\section{Commentary}

Practice in the area of the medical diagnosis of CSA continues to evolve as new observations and experiences are added to our knowledge base. For the past decade, professionals in the field have been working toward agreement as to terminology (for example, the draft guidelines for descriptive terminology proposed by the American Professional Society on the Abuse of Children) and the implication of various physical findings with regard to CSA..$^{1-6}$ Clinicians have gathered in consensus panels to categorise physical findings such as those specific or diagnostic for CSA, those strongly suggestive of CSA, and non-specific abnormalities which may be the results of CSA but are also found in the non-abused population. The quality of data collection has improved considerably with careful application of sound study design aimed at decreasing potential bias: for example, the use of larger study populations, of normal controls for comparison, of panels of experts evaluating colposcopic photographs, and of thorough documentation of CSA independent of the physical findings.

Although a standardised, agreed upon list of normal and abnormal findings is still under development by the American Professional Society on the Abuse of Children, independent studies have arrived at similar conclusions albeit obscured by differences in terminology. 